\title{
Desenvolvimento infantil e sustentabilidade: modelo computacional para representação dos condicionantes do desenvolvimento infantil
}

\author{
Child Development and Sustainability: computational model for the representation of child \\ development conditions
}

\author{
Tainara Piontkoski Maldaner ${ }^{1}$ \\ Bruna Letícia dos Santos ${ }^{2}$ \\ Luciana Vieira Castilho-Weinert ${ }^{3}$ \\ Wagner Rodrigo Weinert ${ }^{4}$
}

\begin{abstract}
Resumo
Este trabalho visa enfatizar de que forma as condições de desenvolvimento na infância repercutem sobre as questões do Desenvolvimento Sustentável, com enfoque principal em estabelecer um modelo que represente o comportamento dos condicionantes do desenvolvimento de crianças de 1 a 12 meses de idade do município de Pontal do Paraná, litoral paranaense. O trabalho consiste em 3 fases: coleta de dados de 1443 crianças e definição de critérios para atraso no desenvolvimento nesta faixa etária, desenvolvimento de uma base de dados on-line, e mineração de dados para reconhecimento de padrões entre os casos de atraso. Como resultados observa-se elevado número de atraso no desenvolvimento em algumas das faixas etárias analisadas. Os achados deste trabalho possibilitam relacionar estes casos com seus respectivos condicionantes socioeconômicos e ambientais, a fim de complementar e ratificar a relevância deste contexto para o desenvolvimento sustentável.
\end{abstract}

Palavras-chave: Infância. Sustentabilidade. Desenvolvimento. Mineração de dados.

\begin{abstract}
This work aims to emphasize the conditions of development in childhood and its relationship with sustainable development. The main focus is on establishing a model that represents the behavior of the constraints of the Development of children from 1 to 12 months old from the municipality of Pontal do Paraná. The work consists of 3 phases: data collection of 1443 children and definition of criteria for delay in development in this age group, development of an online database, and data mining for pattern recognition between cases of delay. As a result, there is a high number of development delay in some of the analyzed age groups. It is expected at the end of the research to relate these cases to their respective socioeconomic and environmental constraints to complement and ratify the relevance of this context for sustainable development.
\end{abstract}

Keywords: Childhood. Sustainability. Development. Data mining.

\footnotetext{
${ }^{1}$ Fisioterapeuta (UFPR). Mestre em Desenvolvimento Territorial Sustentável da Universidade Federal do Paraná (PPGDTS/UFPR). E-mail: tainara.fisioufpr@gmail.com

${ }^{2}$ Fisioterapeuta (UFPR). Mestre em Desenvolvimento Territorial Sustentável da Universidade Federal do Paraná (PPGDTS/UFPR). E-mail: bls.fisio@gmail.com

${ }^{3}$ Doutora em Engenharia Elétrica e Informática Industrial (UTPR). Professora do PPGDTS/UFPR. E-mail: lucianaweinert@gmail.com

${ }^{4}$ Doutor em Engenharia Elétrica e Informática Industrial (UTPR). Professor do Instituto Federal do Paraná. E-mail: wrweinert@gmail.com
} 


\section{Introdução}

Há tempos o debate sobre desenvolvimento está em evidência. Contudo, este tema passou a receber maior ênfase nas últimas décadas, com o colapso dos paradigmas clássicos e teorias tradicionais. Novas abordagens tornaram-se evidentes, pois atrelavam outros significados para o conceito de desenvolvimento, que não exclusivamente o econômico, mas também com o envolvimento das dimensões social e ambiental (COSTA, 2010). A definição mais conhecida sobre desenvolvimento sustentável, consagrada pelo Relatório Brundtland (1987), afirma que o "Desenvolvimento Sustentável é aquele que satisfaz as necessidades do presente sem comprometer a capacidade das futuras gerações em satisfazer suas próprias necessidades” (WCED, 1987).

A falta de atenção às crianças obstrói o direito de milhões delas de adquirir todo o seu potencial de desenvolvimento, além de ser um desperdício devastador de capital humano, por deixar a próxima geração mal equipada para resolver os desafios que travam indivíduos, comunidades e sociedades. Por isso, muitos países aumentam seus investimentos em estratégias para a primeira infância (PI), pois pensa-se que o desenvolvimento das capacidades na PI afeta um indivíduo em todo o curso de sua vida (BRITTO et al., 2016; MARTORELL et al., 2010). Segundo o Relatório de Desenvolvimento Humano, desenvolvido pela Organização das Nações Unidas em 2014, a população mundial de crianças com menos de 5 anos de idade ultrapassa 659 milhões, o que corresponde a cerca de 9,1\% da população total do mundo (PNUD, 2014). Portanto, investimentos substanciais no desenvolvimento das crianças são a base para o desenvolvimento sustentável nos próximos anos (RICHTER et al., 2016; PERSSON, 2017).

O setor da saúde apoia a PI de maneira mais imediata, pelo extenso contato com gestantes, famílias e crianças (RICHTER et al., 2016). Entretanto, entende-se que a saúde é um objeto complexo, e por isso requer um instrumental teórico não mais referenciado à epistemologia positivista e analítica. Ela deve partir de uma perspectiva totalizante, e, portanto, transdisciplinar (BATISTELLA, 2007). Destarte, este trabalho aborda a pesquisa no campo disciplinar da saúde sob a ótica do Desenvolvimento Territorial Sustentável (DTS), definido como um processo que se busca alcançar no longo prazo e cujas consequências não são totalmente previsíveis (HOLLING; BERKES; FOLKES, 1998). Essa definição baseia-se numa noção de sustentabilidade forte, que considera a solidariedade entre as gerações e que atenta para as três dimensões interdependentes do desenvolvimento: a dimensão ecológica, a dimensão social e a dimensão econômica. Neste contexto, apresenta-se como objeto de estudo desta pesquisa a análise dos fatores sociais, econômicos e ambientais que influenciam o desenvolvimento infantil no primeiro ano de vida. 
A via causadora de déficits no desenvolvimento é multifacetada e há um número crescente de crianças a considerar, bem como suas famílias. Os profissionais, os serviços de saúde e os gestores necessitam de ferramentas que mostrem informações imediatas para orientar suas ações (CAESAR et al., 2016). Deste modo, o armazenamento de grande quantidade de dados é uma realidade em cenários que envolvam a saúde da criança, por sua característica multidimensional. Há que se considerar ainda a necessidade de transformação destes dados em informação para que se possa gerar conhecimento sobre a problemática em questão.

Nesta perspectiva, em que se tem uma problemática ampla e que abrange grande quantidade de variáveis em cada uma de suas diferentes dimensões de análise, é necessário realizar processos formais e sistematizados de aquisição do conhecimento e representação do conhecimento. Esta gestão eficiente da informação é o que possibilita gerar inferências e deduções, ou seja, extrair conclusões por meio da visualização de relações que estão implícitas no contexto que se analisa. Para estes processos de inferência utiliza-se processamentos computacionais para gerar conhecimento, mais especificamente a Mineração de Dados (Data Mining), utilizada para a descoberta de informações em meio a grandes quantidades de dados, onde se revela estruturas de conhecimento, a guiar condições de certeza limitada (HAN; KAMBER, 2006).

Portanto, o objetivo deste trabalho é estabelecer um modelo que represente o comportamento dos condicionantes do desenvolvimento de crianças de 1 a 12 meses de idade do município de Pontal do Paraná, litoral do Estado do Paraná, para que auxiliem no processo resolutivo das políticas públicas (PPs), de forma a possibilitar o desenvolvimento sustentável.

\section{Material e Métodos}

Trata-se de um estudo analítico, observacional, transversal (ASLAM et al., 2012), composto por três fases (Figura 1), cuja aprovação ética foi emitida pelo comitê de ética do setor de ciências da saúde da UFPR (CAAE n. ${ }^{\circ}$ 21001613.9.0000.0102). A pesquisa ocorreu no município de Pontal do Paraná, na Unidade de Saúde da Mulher e da Criança, um centro de referência em saúde materno infantil.

Os participantes da pesquisa foram 1443 crianças de ambos os sexos, com idade entre $1 \mathrm{e}$ 12 meses, cujo recrutamento ocorreu por meio do convite a todos os atendidos pela puericultura no SUS, neste município, desde que se enquadrassem nos critérios de exclusão e inclusão deste estudo. Como critérios de inclusão considerou-se estar na faixa etária avaliada, ter o consentimento dos pais ou responsáveis expresso por meio de sua assinatura ao Termo de Consentimento Livre e 
Esclarecido (TCLE), e residir no município de Pontal do Paraná. Como critérios de exclusão definiu-se a presença de patologias ortopédicas ou neurológicas que cursem com alteração no Desenvolvimento Motor Típico, e a utilização de sondas gástricas ou enterais que dificultassem o manuseio durante a avaliação motora.

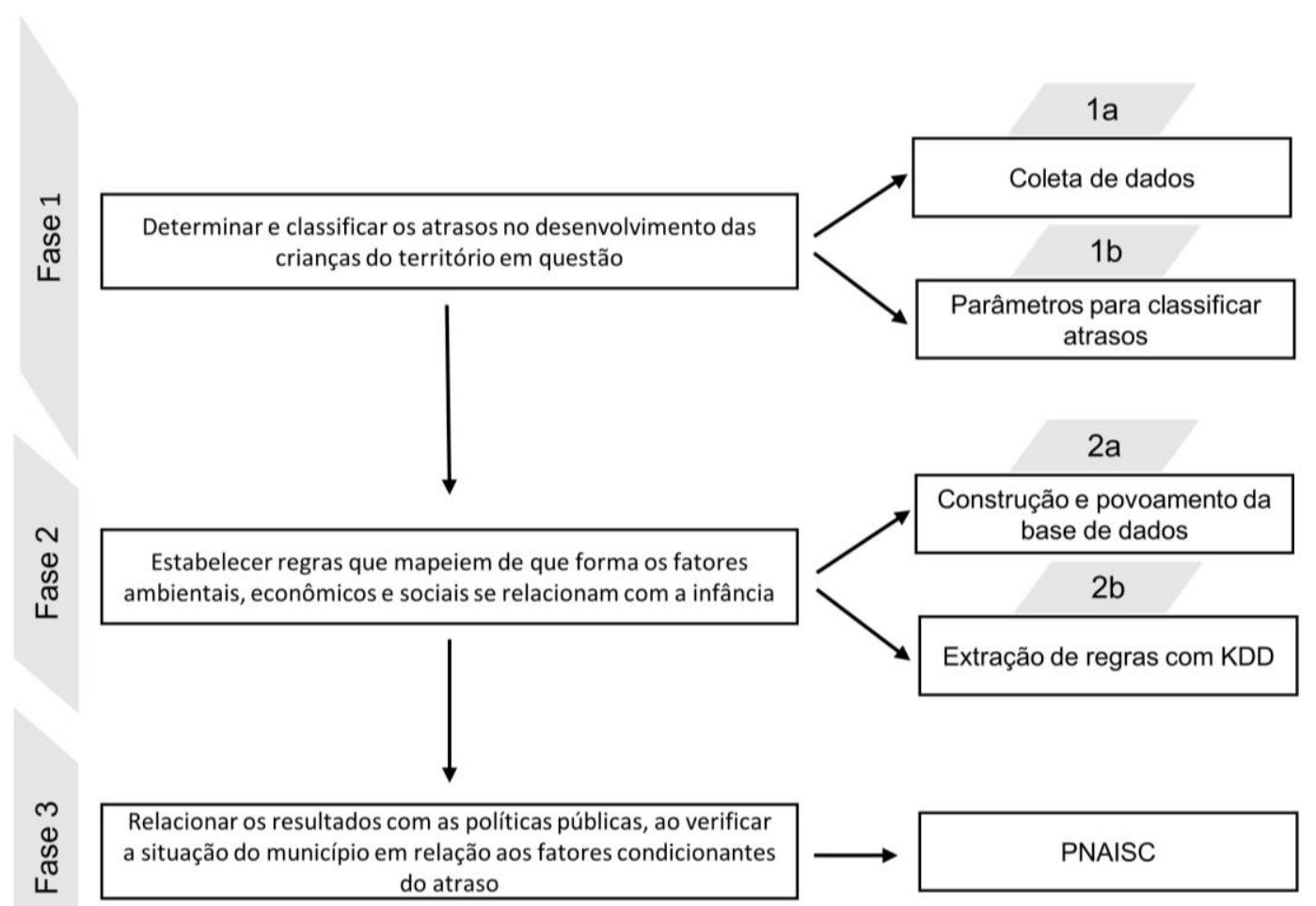

FIGURA 1 - ETAPAS METODOLÓGICAS DA PESQUISA

FONTE: os autores.

Como instrumentos desta pesquisa, utilizou-se um questionário aplicado aos pais e/ou responsáveis, com informações importantes para traçar o perfil das condições sociais, ambientais, econômicas e, consequentemente, de saúde da população deste território (Tabela 1); e um formulário para avaliação motora de Castilho-Weinert, Lopes e Weinert (2015), com enfoque na análise dos componentes do desenvolvimento motor típico por faixa etária (reflexos, reações, habilidades motoras, planos e padrões de movimento). 


\begin{tabular}{|c|c|}
\hline Categoria & Variáveis \\
\hline Dados Pessoais & Nome; nome do responsável; endereço; sexo; data de nascimento. \\
\hline Dados do Parto e Nascimento & $\begin{array}{l}\text { Peso; comprimento; perímetro cefálico; idade gestacional; tipo de parto; parto } \\
\text { hospitalar; notas de Apgar; Internamentos; icterícia. }\end{array}$ \\
\hline Dados Atuais do Bebê & $\begin{array}{l}\text { Intercorrências; peso; comprimento; perímetro cefálico; amamentação exclusiva; } \\
\text { amamentação parcial; calendário de vacinas. }\end{array}$ \\
\hline Dados Maternos & $\begin{array}{l}\text { Idade; } \mathrm{n}^{\circ} \text { de gestações e abortos; intercorrências; vícios; hábitos alimentares; } \mathrm{n}^{\circ} \text { de } \\
\text { consultas no pré-natal; escolaridade. }\end{array}$ \\
\hline Dados Socioeconômicos & Renda familiar; nº de residentes no domicílio; auxílios sociais. \\
\hline Dados Socioambientais & Água encanada; esgoto. \\
\hline
\end{tabular}

FONTE: os autores.

A fase 1a, coleta de dados, ocorreu no período de outubro de 2013 a fevereiro de 2017 . Na fase 1b, estabelecimento dos parâmetros para a classificação dos atrasos no desenvolvimento, utilizou-se os critérios mínimos de habilidade motoras a serem atingidas de acordo com a faixa etária do bebê (Tabela 2) estabelecidos pela Academia Americana de Pediatria (NORITZ, MURPHY and NEUROMOTORSCREENING EXPERT PANEL, 2013). Para a caracterização dos casos de desvio do desenvolvimento motor (DM) típico, a extensão do atraso foi classificada como leve, quando a idade motora era até $33 \%$ abaixo da idade cronológica; moderada, quando a idade motora se situava entre 34-66\% da idade cronológica; e grave, quando a idade motora era $66 \%$ abaixo da idade cronológica (MAJNEMER; SHEVELL, 1995; MCDONALD et al., 2006).

TABELA 2 - CRITÉRIOS PARA ESTABELECIMENTO DE ATRASO

\begin{tabular}{cc}
\hline Mês & Habilidades Avaliadas \\
\hline 2 e 3 & Controle de cabeça parcial \\
4 e 5 & Rolar em bloco; Puppy \\
6,7 e 8 & Rolar dissociado; Sentar não funcional \\
9,10 e 11 & Long sitting (sentar funcional); semi-ajoelhado; engatinhar
\end{tabular}

12

Em pé voluntário; marcha independente

FONTE: Adaptado de NORITZ, G.H.; MURPHY, M.A.; NEUROMOTOR SCREENING EXPERT PANEL (2013).

Na fase 2a, construção e povoamento de uma base de dados online e a inserção, houve a modelagem e desenvolvimento da base de dados. Na modelagem utilizou-se o conceito de banco de 
dados relacional com a ferramenta Workbench e para a implementação o sistema gerenciador de banco de dados MySQL- Na sequência houve o povoamento da base de dados por meio da inserção de todos os dados coletados nas 1443 avaliações realizadas.

A fase $2 b$, extração de regras através do KDD, tem o objetivo de identificar padrões em que se verifique de que forma os casos de atraso no desenvolvimento durante a infância se relacionam com os condicionantes ambientais, econômicos e sociais. Neste processo iniciou-se a simulação de algumas regras de produção que visam identificar possíveis relações entre as dimensões do problema da pesquisa.

$\mathrm{Na}$ fase 3, pretende-se relacionar os resultados das fases anteriores com as PPs, ao verificar a situação do município em relação aos fatores condicionantes do atraso. A reflexão sobre a temática DTS e saúde na infância como base para PPs dar-se-á através de discussões acerca da Política Nacional de Atenção Integral à Saúde da Criança - PNAISC (BRASIL, 2015) no contexto da Atenção Primária à Saúde (BRASIL, 2011), das Redes de Atenção à Saúde (BRASIL, 2010) e dos princípios do SUS, bem como sua implementação no território da pesquisa.

\section{Resultados}

O presente estudo avaliou 1443 crianças situadas na faixa-etária entre 1 e 12 meses. Entre os dados sistematizados, a Tabela 3 apresenta os que são referentes às condições de nascimento da população deste estudo.

TABELA 3 - CONDIÇÕES DE NASCIMENTO

\begin{tabular}{|c|c|c|c|c|}
\hline Variável & Mínimo & Máximo & Média & Desvio Padrão \\
\hline Peso ao nascimento & 950 & 5520 & 3249,5 & $\pm 530,6$ \\
\hline Comprimento ao nascimento & 33 & 62 & 49 & $\pm 12,1$ \\
\hline Perímetro Cefálico ao nascimento & 26,5 & 46 & 34,2 & $\pm 1,9$ \\
\hline Idade Gestacional & 23 & 42 & 38,9 & $\pm 1,5$ \\
\hline Apgar $1^{\circ}$ minuto & 1 & 10 & 8,7 & $\pm 1,2$ \\
\hline Apgar $5^{\circ}$ minuto & 4 & 10 & 9,7 & $\pm 0,5$ \\
\hline
\end{tabular}

FONTE: os autores.

NOTA: Peso em gramas; Comprimento e Perímetro Cefálico em centímetros; Idade Gestacional em semanas.

O Gráfico 1 apresenta a presença de atraso no desenvolvimento da população estudada. Cada criança recebeu a classificação sem atraso, atraso leve, atraso moderado e atraso grave. A Tabela 4 apresenta a classificação de atraso no desenvolvimento segundo a idade. 


\section{GRÁFICO 1- PRESENÇA DE ATRASO NO DESENVOLVIMENTO}

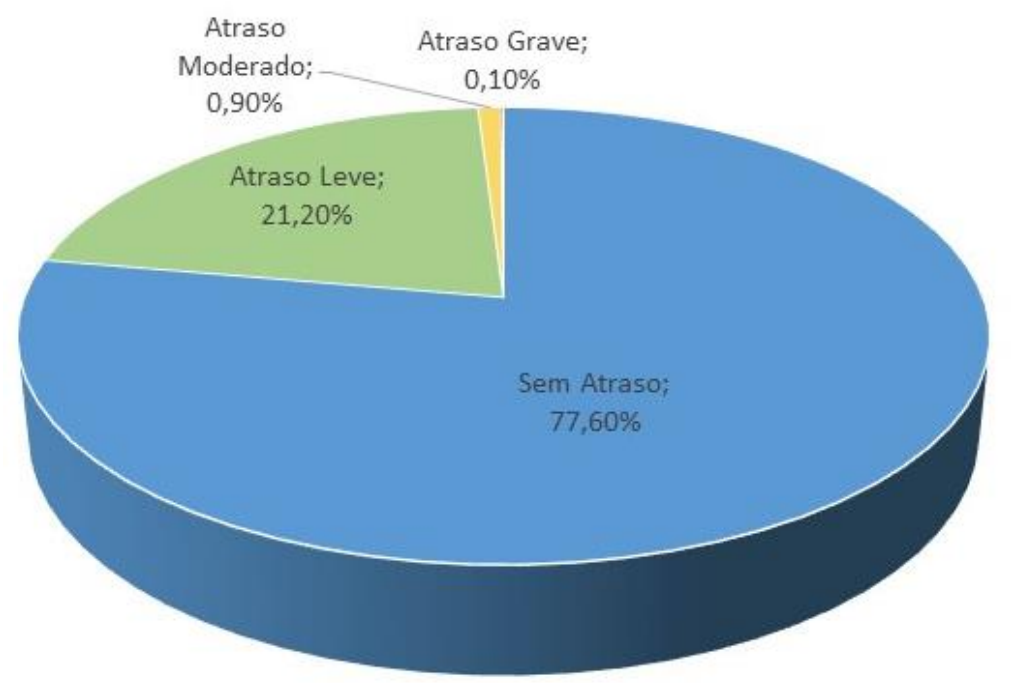

FONTE: os autores.

TABELA 4 - CLASSIFICAÇÃO DE ATRASO NO DESENVOLVIMENTO SEGUNDO A IDADE

\begin{tabular}{ccccccccc}
\hline Mês & \multicolumn{2}{c}{ Sem Atraso } & \multicolumn{2}{c}{ Atraso Leve } & \multicolumn{2}{c}{ Atraso Moderado } & \multicolumn{2}{c}{ Atraso Grave } \\
\hline & $\mathbf{n}$ & $\boldsymbol{\%}$ & $\mathbf{n}$ & $\boldsymbol{\%}$ & $\mathbf{n}$ & $\%$ & $\mathbf{n}$ & $\%$ \\
2 & 298 & 100 & 0 & 0 & 0 & 0 & 0 & 0 \\
3 & 162 & 80,19 & 40 & 19,80 & 0 & 0 & 0 & 0 \\
4 & 137 & 99,27 & 1 & 0,72 & 0 & 0 & 0 & 0 \\
5 & 82 & 49,39 & 84 & 50,60 & 0 & 0 & 0 & 0 \\
6 & 91 & 77,11 & 27 & 22,88 & 0 & 0 & 0 & 0 \\
7 & 61 & 53,50 & 49 & 42,98 & 4 & 3,5 & 0 & 0 \\
8 & 76 & 86,36 & 12 & 13,63 & 0 & 0 & 0 & 0 \\
9 & 74 & 96,10 & 3 & 3,89 & 0 & 0 & 0 & 0 \\
10 & 40 & 48,78 & 40 & 48,78 & 2 & 2,43 & 0 & 0 \\
11 & 45 & 72,58 & 16 & 25,80 & 0 & 0 & 1 & 1,61 \\
12 & 39 & 90,69 & 4 & 9,30 & 0 & 0 & 0 & 0 \\
\hline
\end{tabular}

FONTE: os autores.

O maior percentual de atraso leve encontra-se aos 12 meses (56,6\%), seguido dos 4 meses $(50,6 \%)$ e 9 meses (48,7\%). O atraso moderado ocorre aos 3 (3,5\%), 9 (2,43\%) e 12 meses (1,81\%). O atraso grave ocorre com mais frequência aos 12 meses (14,54\%), seguido dos 10 meses $(1,61 \%)$.

A Tabela 5 apresenta os resultados de renda familiar da população estudada. Destas famílias, 9,3\% recebem algum tipo de benefício social do governo. Em média, o número de residentes em cada família é de 4,3 pessoas. 45,3\% dos domicílios possuem água encanada e, em 
relação ao destino do esgoto, $65 \%$ destinam às fossas; $34 \%$ estão conectados às redes de esgoto e $1 \%$ destinam aos rios da região.

TABELA 5 - RENDA FAMILIAR

\begin{tabular}{cc}
\hline Categoria & $\%$ \\
\hline Até 1 salário mínimo & $27,2 \%$ \\
De 1 a 3 salários mínimos & $55,7 \%$ \\
De 3 a 5 salários mínimos & $15,6 \%$ \\
Mais que 5 salários mínimos & $1,3 \%$ \\
\hline
\end{tabular}

FONTE: os autores.

A Tabela 6 expõe o nível de escolaridade materna da população. Em média, as mães têm 25,4 anos de idade, com uma média de gestações de 2,1 filhos e 0,29 números de abortos.

TABELA 6 - ESCOLARIDADE MATERNA

\begin{tabular}{ccc}
\hline Categoria & $\%$ \\
\hline Analfabeto & 0,2 \\
Ensino Fundamental Incompleto & 15,5 \\
Ensino Fundamental & 25,8 \\
Ensino Médio & 39,5 \\
Graduação & 13,2 \\
Ensino Técnico & 3,4 \\
Pós-Graduação & 2 \\
\hline
\end{tabular}

FONTE: os autores.

\section{Discussão}

\section{Condições de Nascimento}

O acompanhamento do crescimento e desenvolvimento infantil consiste em uma das mais importantes estratégias básicas de saúde no Brasil. Ele ocorre desde a concepção e deve prosseguir, ao menos, durante o primeiro ano de vida da criança (SILVA et al., 2013). Os dados sobre o período gestacional e as condições de nascimento de uma população são importantes informações para a compreensão do desenvolvimento global infantil e, portanto, para o DM. Especialistas em pesquisas em saúde reprodutiva, materna, neonatal e da criança enfatizam que o cuidado à criança deve iniciar ainda antes de seu nascimento e que intervenções durante o período gestacional podem reduzir condições adversas como o nanismo e o baixo peso ao nascer (BLACK et al., 2016). 
As crianças avaliadas neste estudo apresentaram média de peso adequado ao nascimento. Como critérios para classificação do peso ao nascer a Organização Mundial da Saúde (OMS) (WHO, 1996) utiliza: baixo peso (crianças com menos de 2500g), peso insuficiente (2500g a 2999g), peso adequado (3000g a 3999g) e excesso de peso (4000g ou mais) (LIMA; SAMPAIO, 2004). O estudo de Silva, Engstron e Miranda (2015) verificou que o peso ao nascer situava-se entre $1.775 \mathrm{~g}$ e $4.900 \mathrm{~g}$ com média de $3.235 \mathrm{~g}$. No estudo de Souza e colaboradores (2013) a média de peso ao nascer foi de $3.292 \mathrm{~g}$, bem como no estudo de Cagliari e colaboradores (2016), onde verificou-se peso ao nascimento entre $1.510 \mathrm{~g}$ e $5.320 \mathrm{~g}$, com média de $3.415 \mathrm{~g}$. Capelli e colaboradores (2014) obteve média de $3.110 \mathrm{~g}$ de peso ao nascimento. Verifica-se que os resultados de peso ao nascimento destes estudos evidenciam que a maior parte das crianças encontra-se com peso adequado ao nascimento, como mostram as médias apresentadas. No entanto, existem casos tanto de peso insuficiente ao nascimento, quanto de excesso de peso ao nascimento, o que corrobora com os achados deste estudo.

A avaliação do crescimento das crianças foi comparada com a curva padrão de referência mundial do NCHS (NATIONAL CENTER FOR HEALTH STATISTICS, 1977). Para meninos, o NCHS considera comprimento adequado 49,9 e, para meninas, 49,3. Visualiza-se que a média deste estudo apresenta valores próximos da referência da NCHS. Os estudos de Palombo (2014), Carniel (2015), e Ferreira e Barbosa (2017) verificam comprimento ao nascimento entre $46,3 \mathrm{~cm}$ e $47,5 \mathrm{~cm}$, situando-se pouco abaixo do esperado pelas curvas do NCHS.

Para análise dos dados de perímetro cefálico ao nascimento, utiliza-se as curvas de referência da OMS de 2006 (WHO, 2006). As crianças deste estudo encontram-se com mensuração de perímetro cefálico de acordo com o adequado segundo a literatura. Os estudos de Gonçalves e colaboradores (2015), Lima e colaboradores (2014) e Wille, Carbonari e Bottaro (2015) apresentaram média de perímetro cefálico de $34,5 \mathrm{~cm}$, o que corrobora com a literatura e com os achados deste estudo.

A idade gestacional ao nascimento é categorizada em seis grupos: prematuro precoce: $<34$ semanas; prematuro tardio: 34 a $<37$ semanas; termo precoce: 37 a $<39$ semanas; termo: 39 a $<41$ semanas; termo tardio: 41 a $<42$ semanas; e pós-termo: $42<45$ semanas (ACOG's CLINICAL GUIDELINES, 2013; SPONG et al., 2011). A média de idade gestacional do presente estudo situa as crianças na categoria termo precoce. No estudo de Cagliari (2016), visualiza-se crianças entre as categorias de prematuro precoce a pós-termo, o que corrobora parcialmente com os achados desta pesquisa. Estudos atuais enfatizam que a prematuridade é um dos principais fatores de risco biológico para o desenvolvimento (EICKMANN; MALKES; LIMA, 2012; GOMES et al., 2015; MAGGI et al., 2014; SILVA et al., 2016). 
As notas obtidas no Boletim de Apgar deste estudo indicam boas condições de nascimento. Escores de 8 a 10 indicam que o bebê não precisa de extensiva ressuscitação e provavelmente não terá problemas futuros. O escore 7 pode indicar anóxia leve. Um escore de 4 a 6 pode indicar anóxia moderada. Quando o escore é de 0 a 3, significa que a criança está em anóxia grave (APGAR, 1953; CASY; MCINTIRE; LEVENO, 2001; DRAGE; BERENDES, 1966; TECKLIN, 2002). Estes dados corroboram com a não necessidade de utilização de Unidade de Terapia Intensiva neonatal. Estudos realizados no Brasil apresentaram notas de Apgar próximas ao estudo em questão, como o trabalho de Cagliari e colaboradores (2016) e Ferreira e Barbosa (2017). Esta pontuação tem grande importância para o DM, porque condições adversas ocorridas no nascimento resultam em baixos valores de Apgar e demonstram a necessidade de se realizar medidas de intervenção precoce, através de técnicas de estimulação, para prevenção de possíveis sequelas (APGAR, 1953; CASY; MCINTIRE; LEVENO, 2001; TECKLIN, 2002).

A maior parte dos entrevistados neste estudo relatou ter renda entre 1 e 3 salários mínimos, seguido de menos de um salário mínimo. Estes dados corroboram com os achados de Perrone e colaboradores (2015), onde 40\% tinham entre 1 e 3 salários mínimos. Silva, Ferreira e Queiroga (2014) e Borges e Salomão (2015) verificaram que a maior prevalência foi de menos de um salário mínimo.

A presença de água encanada (45,3\%) e destino correto de esgoto (34\%) das residências deste estudo encontram-se aquém do relatado na literatura. Ribeiro, Perosa e Padovani (2014) verificaram que 98,4\% das residências possuem água e encanada, e 78,5\% destinam seus resíduos na rede de esgoto.

A maioria $(65,3 \%)$ das mães questionadas neste estudo concluíram o ensino fundamental e médio. Os resultados de Silva, Ferreira e Queiroga (2014) corroboram parcialmente com estes achados, pois a maior prevalência foi de ensino fundamental incompleto (40\%), seguido do ensino fundamental completo (25\%) e ensino médio completo (25\%). Cavalcanti e colaboradores (2015) também encontraram resultados semelhantes, onde $41 \%$ da população estudou de 5 a 8 anos, e $34 \%$ estudou menos do que 4 anos.

No presente estudo, o perfil etário das mulheres investigadas apresenta-se adequado $(25,4$ anos), pois corrobora com dados de outros estudos recentes. Carvalhaes e colaboradores (2013), por exemplo, verificaram que a maioria dos atendimentos às gestantes na Atenção Primária à Saúde (90\%) incluíam mulheres entre 18 e 34 anos de idade, e se confirma também pelo estudo de perfil epidemiológico de Silva e colaboradores (2015) em mulheres do Tocantins.

No Brasil, a taxa total de fecundidade reduziu substancialmente nas últimas décadas. Em 1960, as mulheres tinham cerca de 6,3 filhos. Já de 2002 a 2006 esta taxa caiu para 1,8 filhos por 
mulher, valor próximo ao obtido neste estudo. Os dados de Pontal do Paraná divergem daqueles encontrados em outros estudos no que diz respeito ao número de gestações ( 2,1 filhos). Enquanto no território estudado a maioria das gestantes refere estar na segunda gestação, estudos relatam maior frequência de primíparas (CARVALHAES et al., 2013; WEIDLE et al. 2014; SILVA et al., 2015). Nogueira e colaboradores (2011) também obtiveram maior prevalência de secundigestas $(40 \%)$.

\section{Desenvolvimento Motor e Atraso}

O perfil detalhado sobre o DM na primeira infância viabiliza a identificação precoce de alterações que possam culminar em comprometimentos significativos em outras dimensões do desenvolvimento a longo prazo, inclusive na cognição. Esta identificação orienta a adoção de condutas de estimulação precoce, a fim de reverter ou minimizar este quadro (CASTILHOWIENERT; LOPES; WEINERT, 2015).

No presente estudo, verificou-se a presença de atraso leve do $2^{\circ}$ ao $12^{\circ}$ mês, em alguns casos em mais de 50\% das avaliações. Este percentual é muito superior aos valores encontrados em outros estudos. Em estudo realizado no Acre, Estado do norte do país, 13\% dos bebês menores de apresentaram suspeita de atraso motor (ANDRADE; NEGREIROS, 2013). Já no Nordeste, na capital do Ceará, 9\% nascidos dos nascidos a termo e 3,8\% dos prematuros apresentaram atraso ou risco de atraso no desenvolvimento motor (LOPES; CARDOSO, 2014).

Em um estudo comparativo entre os sexos com a utilização da Alberta Infant Motor Scale (AIMS), Venturella e colaboradores (2013) verificaram que a suspeita de atraso esteve presente em $25,6 \%$ dos casos e a condição de atraso em apenas 1,1\%. Pereira, Saccani e Valentini (2016) obtiveram resultados semelhantes com bebês de 2 a 16 meses, avaliados pela AIMS e pela Bayley Scale of Infant Development em três momentos distintos. De acordo com a AIMS, verificou-se o atraso de 2 a 6,1\%, a suspeita de atraso de 22,4 a 24,5\%; e o DM normal de 71,4 a 75,5\%. A escala Bayley não apontou nenhuma criança com atraso significante. Contudo, observou-se o atraso leve de 6,1 a $12,2 \%$ da amostra.

Estas divergências nos resultados dos atrasos podem ocorrer pois não existe um único referencial para classificação dos atrasos no desenvolvimento infantil, e habitualmente cada escala de avaliação possui uma graduação própria para a determinação desta variável. Também existem muitas discussões a respeito do significado do termo atraso, e os autores podem atribuir diferentes significados à palavra (DORNELAS; DUARTE; MAGALHÃES, 2015).

O estudo de Saccani e Valentini (2010) mostra casos de atraso principalmente a partir do segundo semestre vida, onde o bebê diminui substancialmente a atividade reflexa primitiva e 
intensifica a motricidade voluntária ampla (FORMIGA, 2015). Da mesma forma, Pereira, Saccani e Valentini (2016), em um estudo longitudinal, verificaram maior prevalência de atrasos no terceiro quadrimestre de vida. Por outro lado, em um estudo de 2010, estes mesmos autores observaram maior incidência de atraso no primeiro trimestre. Sugere-se que a variabilidade destes resultados pode relacionar-se a questões de individualidade ou a períodos de estabilidade nas aquisições motoras (SACANNI; VALENTINI, 2010).

A estimulação precoce do DM torna-se fundamental para que os atrasos não tomem caráter definitivo e atrapalhem outros domínios de desenvolvimento da criança. Pois, salienta-se que o DM possui importante papel devido às influências que exerce sobre as habilidades cognitivas e a capacidade de aprendizagem escolar da criança (MANCINI et al., 2004).

\section{Considerações Finais}

Esta pesquisa demonstrou o perfil do desenvolvimento infantil do ponto de vista da saúde com ênfase na motricidade, e os fatores ambientais, econômicos e sociais. As crianças apresentaram boas condições de vitalidade ao nascimento, e dados maternos e socioeconômicos que corroboram com os achados da literatura. No entanto, os dados socioambientais encontram-se aquém do esperado. A maioria dos casos observados de atraso foram classificados como leve. Tal fato deve-se ao constante acompanhamento das crianças no programa de puericultura, bem como a implementação das políticas públicas de atenção integral à saúde da criança.

A realização deste trabalho resulta da necessidade de se aproximar de um tema pouco explorado, ou quase inédito, ao escopo de estudos do DTS: saúde e desenvolvimento infantil. Tal temática estrutura-se na perspectiva de que indivíduos saudáveis, esclarecidos e socialmente capazes são agentes promotores do desenvolvimento socioeconômico, cultural e ambiental. Faz-se necessário que os indivíduos exerçam todos os seus direitos fundamentais, o que viabilizará outra forma de vida em sociedade na qual seja possível gerar e adotar meios de subsistência, direitos e oportunidades, ou seja, para o alcance dos objetivos de um novo modelo de desenvolvimento com vistas à sustentabilidade.

Neste sentido, destaca-se a importância de estudos que abordem as temáticas de saúde infantil e DTS, pois acredita-se que crianças com níveis adequados de desenvolvimento tornem-se agentes transformadores em um ambiente sustentável, pois a saúde de um indivíduo em cada fase de sua vida afetará a saúde nas outras fases, com efeitos cumulativos para a próxima geração. Como 
continuidades deste estudo, pretende-se relacionar as condições de desenvolvimento infantil com as dimensões social, econômica e ambiental do desenvolvimento sustentável.

\section{Referências}

ACOG's CLINICAL GUIDELINES. Medically indicated late-preterm and early-term deliveries. Committee Opinion No. 560. American College of Obstetricians and Gynecologists. Obstet Gynecol, v.121, p.908-10, 2013.

ANDRADE, J.L.; NEGREIROS, M.M. Suspected of neuropsychomotor developmental delay in children under one year assisted by a family health unit of Rio Branco (Acre). Rev. APS, v.16, n.1, 2013.

APGAR, V. A proposal for a new method of evaluation of the newborn infant. Curr Res Anaesth Anal, v.32, p.260-7, 1953.

ASLAM, S.; GEORGIEV, H.; MEHTA, K.; KUMAR, A. Matching research design to clinical research questions. Indian. J. Sex. Transm. Dis., v.33, p. 49- 53, 2012.

BATISTELLA, C. Abordagens contemporâneas do conceito de saúde. In: FONSECA, A.F.; CORBO, M.B. O território e o processo saúde-doença. Rio de Janeiro: EPSJV/Fiocruz, 2007.

BLACK, M.M.; WALKER, S.P.; FERNALD, L.C.H.; ANDERSEN, C.T.; DIGIROLAMO, A.M.; LU, C.; MCCOY, D,C,. FINK, G.; SHAWAR, Y.R.; SHIFFMAN, J.; DEVERCELLI, A.E.; WODON, Q.T.; VARGAS-BARÓN, E.; GRANTHAM-MCGREGOR, S. Early childhood development coming of age: science through the life course. The Lancet, v.389, n.10064, p.77-90, 2016.

BORGES, L.C.; SALOMÃO, N.M.R. Concepções do desenvolvimento infantil e metas de socialização maternas em contexto não urbano. Estudos de Psicologia, v.20, n.2, p.114-125, 2015.

BRASIL. Portaria GM n ${ }^{\circ}$ 1130, de 5 de agosto de 2015. Institui a Política Nacional de Atenção Integral à Saúde da Criança (PNAISC) no âmbito do Sistema Único de Saúde (SUS). Diário Oficial da União, 2015.

BRASIL. Portaria $n^{\circ}$ 2.488, de 21 de outubro de2011. Aprova a Política Nacional de Atenção Básica, estabelecendo a revisão de diretrizes e normas para a organização da Atenção Básica, para a Estratégia Saúde da Família (ESF) e o Programa de Agentes Comunitários de Saúde (PACS). Diário Oficial da União, 2011.

BRASIL. Portaria $n^{\circ} 4.279$, de 30 de dezembro de 2010. Estabelece diretrizes para a organização da Rede de Atenção à Saúde no âmbito do Sistema Único de Saúde (SUS). Diário Oficial da União, 2010.

BRITTO, P.R.; LYE, S.; POULX, K.; YOUSAFZAI, A.K.; MATTHEWS, S.G.; VAIVADA, T.; PEREZ-ESCAMILLA, R.; RAO, N.; IP, P.; FERNALD, C.H.; MACMILAN,, H.; HANSON, M.; WACHS, T.D.; YAO, H.; YOSHIKAWA, H.; CEREZO, A.; LECKMAN, J.F.; BHUTTA, Z and 
theEarly Childhood Development Interventions Review Group. Nurturing care: promoting early childhood development. The Lancet, v.389, n.10064, p.91-102, 2016.

CAESAR, R.; BOYD, R.N.; COLDITZ, P.; CIONI, G.; WARE, R.S.; SALTHHOUSE, K.; DOHERTY, J.; JACKSON, M.; MATTHEWS, L.; HURLEY, T.; MOROSINI, A.; THOMAS, C.; CAMADOO, L.; BAER, E. Early prediction of typical outcome and mild developmental delay for prioritisation of service delivery for very preterm and very low birthweight infants: a study protocol. BMJ Open, v.6, n.e010726, 2016.

CAGLIARI, P. Z.; VERAS, T.N.V.; VIEIRA, C.E.F.; BERTELLI, L.J.; RAMOS, M.C. Alterações detectadas pelo teste do reflexo vermelho. Arquivos Catarinenses de Medicina, v. 45, n. 3, 2016.

CAPELLI, J. C. S. PONTES, J.S.; PEREIRA, S.E.A.; SILVA, A.A.M.; CARMO, C.N.; BOCCOLINI, CRISTIANO SIQUEIRA, ALMEIDA, M.F.L. Peso ao nascer e fatores associados ao período pré-natal: um estudo transversal em hospital maternidade de referência. Cien Saude Colet, v. 19, n.7, p.2063-2072, 2014.

CARNIEL, M. P. SANTETTI, D.; ANDRADE, J.S.; FAVERO, B.P.; MOSCHEN, T.; CAMPOS, P.A.; GOLDANI, H.A.S.; DORNELLES, C.T.L. Validação de um questionário de avaliação nutricional subjetiva global. J Pediatr, p. 596-602, 2015.

CARVALHAES, M.A.B.L; MARTINIANO, A.C.A.; MALTA, M.B.; TAKITO, M.Y.; BENÍCIO, M.H.D. Atividade física em gestantes assistidas na atenção primaria a saúde. Rev Saude Publica; v.47, n.5, p.958-967, 2013.

CASTILHO-WEINERT, L.V.; LOPES, H.S.; WEINERT, W.R. Desenvolvimento motor típico no primeiro ano de vida: caracterização e detalhamento. Fisioterapia Ser, v. 9, n. 1, p. 1- 5, 2015.

CASY, B.M; MC INTERE, D.D; LEVENO, K.J. The continuing value of the Apgar score for the assessment of newborn infants. N Engl J Med, v.334, n.7, p. 467-71, 2001.

CAVALCANTI, S.H. CAMINHA, M.F.C.; FIGUEIROA, J.N.; SERVA, V.M.S.B.D.; CRUZ, R.S.B.L.C.; LIRA, P.I.C.; BATISTA FILHO, M. Fatores associados à prática do aleitamento materno exclusivo por pelo menos seis meses no estado de Pernambuco. Rev. bras. epidemiol., São Paulo, v. 18, n. 1, p. 208-219, mar. 2015.

COSTA, A.A.V.M.R. Agricultura sustentável I: conceitos. Rev. de Ciências Agrárias, Lisboa, v. 33, n. 2, p. 61-74, 2010.

DORNELAS, L.F.; DUARTE, N.M.C.; MAGALHÃES, L.C. Atraso no desenvolvimento neuropsicomotor: mapa conceitual, definições, usos e limitações do termo. Rev. Paulista de Pediatria, v.33, n.1, p.88-

DRAGE, J.S; BARENDES, H. Apgar scores and outcome of the newborn. Pediatr Clin North Am. 13:635, 1966.

EICKMANN, S.H.; MALKES, N.F.A.; LIMA, M.C. Psychomotor development of preterm infants aged 6 to 12 months. Sao Paulo Med. J., v. 130, n. 5, p.299-306, 2012. 
FERREIRA, M. T. S.; BARBOSA, M. U. F. Contribuição da fisioterapia no desenvolvimento motor de uma criança com hidrocefalia: estudo de caso. Encontro de Extensão, Docência e Iniciação Científica (EEDIC), v. 3, n. 1, 2017.

FORMIGA, C.K.M.R. Crescimento e desenvolvimento neuropsicomotor de crianças de zero a dois anos. Pediatria Moderna, v.51, n.11, p.405-410, 2015.

GOMES, E.L.F.D.; PEREIRA, L.C.; VIVIANE, A.G.; BOTTCHER, S.M.; VIRGÍNIA, L.; LIMA, C.; MOURA, R.C.F. Desenvolvimento Motor de RN prematuros. Pediatria Moderna, v.51, n.5, p.168-172, 2015.

GONÇALVES, F. C. L. S. P; LIRA, P. I. C ; EICKMANN, S. H.; LIMA, M.C. Razão peso/PC ao nascer na avaliação do crescimento fetal. Cad. Saúde Pública. Rio de Janeiro, v.31, n.9, p.19952004, 2015.

HAN, J.; KAMBER, M. Data Mining: Concepts and Techniques. 2 ed. The Morgan Kaufmann Series in Data Management Systems, Jim Gray, Series Editor Morgan Kaufmann Publishers, March 2006.

HOLLING, C.; BERKES, F.; FOLKES, C. Ciência, sustentabilidade e gestão de recursos. In: BERKES, F.; FOLKES, C. Linkin Social and ecologycal systems. Cambridge: Cambridge University Press, 1998.

LIMA, P. A; CARVALHO, M; COSTA, A. C; MOREIRA, M. E. Variables associasted with extra uterine growth restriction in very low birth weight infants. J pediatr. Rio de Janeiro, v.90; p.27-7. 2014.

LIMA, G.S.P.; SAMPAIO, H.A.C. Influência dos fatores obstétricos, socioeconômicos e nutricionais da gestante sobre o peso do recém-nascido: estudo realizado em uma maternidade em Teresina, Piaui. Rev. Bras. Saúde Mater. Infant., v.4, n.3, 2004.

LOPES, M.M.C.O.; CARDOSO, M.V.L.M..L. Avaliação do desenvolvimento neuromotor da criança por meio do Harris Infant Neuromotor Test. Rev. Esc Enferm USP, v.48, n.4, p.586-92, 2014.

MAGGI, E.F.; MAGALHAES, L.C.; CAMPOS, A.; BOUZADA, M.C.F. Crianças pré-termo apresentam desempenho motor, cognitivo e funcional desfavorável em relação a neonatos a termo em idade pré-escolar. J. Pediatr. (Rio J.), Porto Alegre, v. 90, n. 4, p. 377-383, 2014.

MAJNEMER, A.; SHEVELL, M.L. Diagnostic yield of the neurologic assessment of the developmentally delayed child. J. Pediatrics, v.127, n.2, p.193-199, 1995.

MANCINI, M.C., BRANDÃO, M.B.; MELO, A.P.P.; SAMPAIO, R.F. Efeito Moderador do risco social na relação entre o risco biológico e desempenho funcional infantil. Rev. Bras. Saúde Mater. Infant., Recife, v.4, n.1, 2004.

MARTORELL, R.; HORTA, B.L.; ADAIR, L.S.; STEIN, A.D.; RICHTER, L.; FALL, C.H.; BHARGAVA, S.K.; BISWAS, S.K.; PEREZ, L.; BARROS, F.C.; VICTORA, C.G.; and Consortium on Health Orientated Research in Transitional Societies Group. Weight gain in the first 
two years of life is an important predictor of schooling outcomes in pooled analyses from five birth cohorts from low- and middle-income countries. J Nutr, v.140, p.348-54, 2010.

MCDONALD, L.; RENNIE, A.; TOLMIE, J.; GALLOWAY, P.; MCWILLIAM, R. Investigation of global developmental delay. Archs Dis Child, v.91, p.701-705, 2006.

NATIONAL CENTER FOR HEALTH STATISTICS. NCHS Growth curves for children, birth-18 years. United States: Vital and Health Statistics, v.11, n.165, p.1-74, 1977.

NOGUEIRA, A.I. SANTOS, J.S.S.; SANTOS, L.L.B.; SALOMON, I.M.M.; ABRANTES, M.M.; AGUIAR, R.A.L.P. Diabetes Gestacional: perfil e evolução de um grupo de pacientes do Hospital das Clínicas da UFMG. Rev Med Minas Gerais, v.21, n.1, p.32-41, 2011.

NORITZ, G.H.; MURPHY, N.A.; NEUROMOTOR SCREENING EXPERT PANEL. Motor Delays, early identification and evaliation. Pediatrics, v.131, n.6, p.2016-2027, 2013.

PALOMBO, C. N. T.; DUARTE, L.S.; FUJIMORI, E.; TORIYAMA, A.T.M. Use and records of child health handbook focused on growth and development. Revista da Escola de Enfermagem da USP, v. 48, n. SPE, p. 59-66, 2014.

PEREIRA, K.R.G.; SACCANI, R.; VALENTINI, N.C. Cognição e ambiente são preditores do desenvolvimento motor de bebês ao longo do tempo. Fisioter. Pesqui., São Paulo, v. 23, n. 1, p. 5967, 2016.

PERRONE, A.C.L.; FIGUEIREDO, G.L.P.; AMARAL, J.H.; SOUZA, C.S.M. Desenvolvimento infantil no interior do Amazonas: avaliação antropométrica de escolares de 9 anos. Sau. \& Transf. Soc., Florianópolis, v.6, n.3, p.42.-09, 2015.

PERSSON, L.A. Prenatal nutrition, socioenvironmental conditions, and child development. Lancet Global Health, p. e127-e128, 2017.

PROGRAMA DAS NACÕES UNIDAS PARA O DESENVOLVIMENTO (2014). Relatório do Desenvolvimento Humano 2014. Sustentar o Progresso Humano: Reduzir as Vulnerabilidades e Reforçar a Resiliência. Portugal: PNUD, 2014.

RIBEIRO, D.G.; PEROSA, G.B.; PADOVANI, F.H.P. Fatores de risco para o desenvolvimento de crianças atendidas em Unidades de Saúde da Família, ao final do primeiro ano de vida:aspectos sociodemográficos e de saúde mental maternal. Ciência e Saúde Coletiva, v.19, n.1, p.215-226, 2014.

RICHTER, L.M.; DAELMANS, B.; LOMBARDI, J.; Heymann, J.; BOO, F.L.; BEHRMAN, J.R.; LU, C.; LUCAS, J.E.; PEREZ-ESCAMILA, R.P.; DUA, T.; BHUTTA, Z.A.; STENBERG, K.; GERTLER, P.; DARMSTADT, G.L. Investing in the foundation of sustainable development: pathways to scale up for early childhood development. The Lancet, v.389, n.10064, p.103-118, 2017.

SACCANI, R.; VALENTINI, N.C. Análise do desenvolvimento motor de crianças de zero a 18 meses de idade: representatividade dos itens da Alberta Infant Motor Scale por faixa etária e postura. Rev. Bras. Crescimento Desenvolvimento Humano, v.20, n.3, p.711-722, 2010. 
SILVA, Â. C. D; ENGSTRON, E. M; MIRANDA, C. T. Fatores associados ao desenvolvimento neuropsicomotor em crianças de 6-18 meses de vida inseridas em creches públicas do Município de João Pessoa, Paraíba, Brasil. Cad. Saúde Pública, Rio de Janeiro, v.31, n.9, p.1881-1893, set, 2015.

SILVA, A.C.F.; FERREIRA, A.A.; QUEIROGA, B.A.M. Desenvolvimento da narrativa oral e o nível de escolaridade materna. Rev. CEFAC., v.16, n.1, p.174-186, 2014.

SILVA, L.P.; MAIA, P.C.; LOPES, M.M.C.O.; CARDOSO, M.V.L.M.L. Confiabilidade intraclasse da Alberta Infant Motor Scale na versão brasileira. Rev Esc Enferm USP, v.41, n.5, p.1046-51, 2013.

SILVA, J.K.M.; SARGI, A.M.; ANDRADE, I.C.O.; ARAUJO, C.C.; ANTONIO, T.D. Motor development of preterm and term infants in the fundamental movement phase: a cross-sectional study. Fisioter. mov, Curitiba, v. 29, n. 3, p. 581-588, 2016.

SOUZA, M. A. C. A.; GUIMARAES, I.C.B.; DALTRO, C.; GUIMARAES, A.C. Associação entre peso de nascimento e fatores de risco cardiovascular em adolescentes. Arq. Bras. Cardiol, v. 101, n. 1, p. 9-17, 2013.

SPONG CY, MERCER BM, D'ALTON M, KILPATRICK S, BLACKWELL S, SAADE G. Timing of indicated late-preterm and early-term birth. Obstet Gynecol, v.118 (2 Pt 1), p.323-33, 2011.

TECKLIN, J.S. Fisioterapia pediátrica. Porto Alegre: Artmed; 2002.

VENTURELLA, C.B.; ZANANDREA, G.; SACCANI, R.; VALENTINI, N.C. Desenvolvimento motor de crianças entre 0 e 18 meses de idade: diferenças entre os sexos. Rev. Motricidade, v.9, n.2, p.3-12, 2013.

WORLD COMISSION ON ENRIRONMENT e DEVELOPMENT - WCED. Our common future. Oxford: Oxford University Press, 1987.

WEIDLE, W.G.; MEDEIROS, C.R.G.; GRAVE, M.T.Q.; BOSCO, S.M.D. Escolha da via de parto pela mulher: autonomia ou indução?. Cad. saúde colet., v.22, n.1, p.46-53, 2014.

WILlE, P. T; CARBONARI, V. Z; BOTTARO, S. M. Avaliação do perímetro cefálico de préescolares de um município do noroeste do Rio Grande do Sul. Salão do Conhecimento, v.1, n.1, 2015.

WORLD HEALTH ORGANIZATION. Care in normal birth: a practical guide. 1996.

WORLD HEALTH ORGANIZATION (2006). [homepage on the Internet]. The WHO child growth standards [cited 2007 Jun 8]. Available from: wttp://www.who.int/childgrowth/ standards/en/ Acesso em: 13 de maio de 2017.

Artigo recebido em 11/04/2018. Aceito para publicação em 15/05/2018. 